\title{
Reducing antibiotic prescribing in Australian general practice: time for a national strategy
}

\author{
Christopher B Del Mar', Anna Mae Scott', Paul P Glasziou', Tammy Hoffmann', Mieke L van Driel ${ }^{\top}$, Elaine Beller', \\ Susan M Phillips ${ }^{3}$, Jonathan Dartnell ${ }^{4}$
}

n April 2017, the Centre for Research Excellence in Minimising Antibiotic Resistance from Acute Respiratory Infections in primary care hosted a national roundtable meeting. Australian researchers, policy makers and organisations came together to discuss ways of dealing with the antibiotic resistance crisis from a general practice perspective. This narrative review pools the literature used during the discussions and a literature search using PubMed (online Appendix).

The antibiotic resistance crisis will have two substantial consequences. The first one has begun: about 1600 people in Australia die directly from antibiotic resistance annually, extrapolating from overseas data. ${ }^{1}$ It will get steadily worse until 2050, when deaths from currently treatable infections will overtake total cancer deaths. The second consequence - yet to arrive - is the risk that antibiotic resistance will pose to now routine high technology medical care. Without reliable antibiotic prophylaxis, procedures such as chemotherapy, bone marrow transplant, much major surgery (eg, joint prostheses) and invasive diagnostic procedures (eg, cardiac catheterisation) will become too dangerous to perform. Many aspects of medical care will retreat into the pre-antibiotic era of the 1930s, the economic consequences of which are catastrophic and have prompted the Chief Medical Officer of the United Kingdom to declare this threat every bit as serious as terrorism. ${ }^{2}$ Governments are attempting to provide incentives for the pharmaceutical industry to generate new classes of antibiotics, ${ }^{2}$ but action aimed at extending the life of the existing antibiotics by conserving their use is equally important.

This crisis is not directly obvious to GPs working in the community. Yet, GPs contribute to most of the antibiotic tonnage consumed by humans in Australia $^{3}$ - exact data are not available in Australia, but in Denmark and Sweden (low community antibiotic-prescribing countries), 90\% of antibiotics for human use are prescribed outside hospitals, and 65\% are prescribed by GPs. ${ }^{4,5}$ Reducing antibiotic usage allows resistance to dissipate, ${ }^{6}$ because the unnecessary metabolic load of metabolising resistance genes in the absence of antibiotics puts these organisms at a selective disadvantage. Some areas of primary care are ripe for reducing antibiotics use, especially prescribing for common acute respiratory infections (ARIs), for which a wealth of evidence shows that the benefits of antibiotics are small and that not using them is generally safe. Other countries use less than half the quantity ${ }^{3}$ with no increase in serious infections. $^{5}$

Gains cannot be achieved quickly or simply. Countries that have achieved great gains in reducing their prescribing rates have done so using multiple interventions in concert and persisting over many years.

The aim of this narrative review is to describe interventions that, if implemented on a national scale and successfully lowered the volume of antibiotics prescribed in general

\section{Summary}

- In Australia, the antibiotic resistance crisis may be partly alleviated by reducing antibiotic use in general practice, which has relatively high prescribing rates - antibiotics are mostly prescribed for acute respiratory infections, for which they provide only minor benefits.

- Current surveillance is inadequate for monitoring community antibiotic resistance rates, prescribing rates by indication, and serious complications of acute respiratory infections (which antibiotic use earlier in the infection may have averted), making target setting difficult.

- Categories of interventions that may support general practitioners to reduce prescribing antibiotics are: regulatory (eg, changing the default to "no repeats" in electronic prescribing, changing the packaging of antibiotics to facilitate tailored amounts of antibiotics for the right indication and restricting access to prescribing selected antibiotics to conserve them), externally administered (eg, academic detailing and audit and feedback on total antibiotic use for individual GPs), interventions that GPs can individually implement (eg, delayed prescribing, shared decision making, public declarations in the practice about conserving antibiotics, and self-administered audit), supporting GPs' access to near-patient diagnostic testing, and public awareness campaigns.

- Many unanswered clinical research questions remain, including research into optimal implementation methods.

- Reducing antibiotic use in Australian general practice will require a range of approaches (with various intervention categories), a sustained effort over many years and a commitment of appropriate resources and support.

practice for ARIs, should reduce community-acquired antibiotic resistance.

\section{Reasons for overprescribing}

There are several reasons why GPs overprescribe for ARIs. ${ }^{7}$ It is difficult - and often impossible - to separate apparently innocuous ARIs from the early stages of very serious ones, ${ }^{8}$ such as community-acquired pneumonia, mastoiditis, epiglottitis or even meningitis, and GPs tend to play it safe. GPs value the doctorpatient relationship and may assume that not prescribing antibiotics threatens this relationship. ${ }^{9}$ Moreover, time-poor GPs may perceive that it is quicker to finish a consultation for ARI with an antibiotic prescription. ${ }^{9}$ There may be also financial concerns, such as not wanting to lose a patient. In addition, clinicians may have misperceptions that may inflate the benefits of medical treatments generally.

\section{Community surveillance and targets}

If we are to reduce antibiotic prescribing, a question arises: reduce to what level? However, before setting targets, we need to decide 
which outcomes to monitor through surveillance. There are three potential main sets of data.

First, we need to know and monitor the background level of antibiotic resistance in the community — reducing this level being the primary objective. The main sources of resistance in patients being admitted to hospital, once originating from hospitals themselves, now originate as often from the community. ${ }^{11}$ The problem is that this community-originated resistance is only currently being monitored passively, by collation of clinically submitted data. ${ }^{3}$ This type of resistance represents only a small proportion of potential infections, since most respiratory infections in general practice (eg, sore throat) are treated without testing ${ }^{12}$ and are, therefore, likely to be heavily distorted towards more complicated or recalcitrant, and hence resistant, specimens. We propose that resistance levels in the community could be monitored using sentinel general practices to systematically sample infections or even uninfected attending patients. Routine monitoring resistance in aerobes (collected by nasal swabs) should be straightforward - although anaerobes (collected by faecal swabs) would be more difficult.

Second, we need to monitor the rates of total antibiotic prescribing, which is the main behaviour that needs to change, even if the focus is on ARIs. Some data are collected by the Drug Utilisation Sub Committee of the Pharmaceutical Benefits Scheme (PBS), but they do not provide clinical indications for antibiotic use, which must come from GPs themselves. Until now, the Bettering the Evaluation and Care of Health program (with a rolling survey of a national sample of $1000 \mathrm{GPs}$ ' clinical activities) gathered data on antibiotic use from GPs, but has just been defunded. ${ }^{13}$ An alternative, sampled less representatively, comes from GP registrars in training, ${ }^{14}$ and a longitudinal GP data collection for the MedicineInsight program ${ }^{15}$ may provide such data in future.

Finally, it is important that we monitor patient safety indicators, such as serious infections not treated with antibiotics when they should have been. Reporting hospital admissions for serious ARIs would achieve this patient safety control, which is done routinely in some countries. ${ }^{4,5}$ But in Australia, this monitoring would require setting up from relevant hospital discharge separation data.

The next step is to set targets. Community resistance targets will have to await baseline data; however, overall antibiotic prescribing targets could be set. These targets are likely to be controversial and may be unwelcomed by GPs worrying about safety. They could be set with reference to international best practice (the prescription rate in Australia is about double that of, for example, the Netherlands), ${ }^{3}$ but recent data show that, currently, GPs prescribe $6-9$ times the rates indicated by guidelines. ${ }^{16}$ This prescription rate suggests the enormous scope for safely reducing prescribing by using the set targets without resorting to normative data.

Interventions to support GPs to reduce their antibiotic prescribing

\section{Regulatory interventions}

Some interventions could produce rapid results. Many GPs' electronic health records default to repeat prescribing to the maximum allowable under the PBS, which may have unintended consequences, such as sending a message to patients that another course of antibiotics is necessary or routine, or leaving an unused repeat prescription (or even more antibiotics) available for use for another illness somewhere in the community. This issue could be redressed either by mandating GP software defaults to "no repeat" prescriptions of antibiotics (with an option to override when clinically indicated), or by changing the PBS criteria and restrictions.

Another action that the Australian Government could implement quickly is to restrict access to several antibiotics earmarked for special conservation, in order to reserve them. Australia has a success story in the lack of resistance to quinolones, which, because they are used little, remain relatively resistance-free compared with other countries. ${ }^{17}$ The mechanism for implementing this restriction is the Authority Prescribing System, in which an extra administrative step requires confirmation of a pre-set diagnostic requirement.

More intractable problems include the dissonance between guideline-suggested durations of antibiotic therapy and the pack size available on the PBS dispensed by pharmacists. ${ }^{18}$ For example, for a child with acute otitis media requiring antibiotics, the duration recommended by the Therapeutic Guidelines ${ }^{19}$ (ie, 5 days) means that a one-year-old child who weighs $10 \mathrm{~kg}$ and is prescribed a $100 \mathrm{~mL}$ bottle of $25 \mathrm{mg} / 5 \mathrm{~mL}$ of amoxicillin is left with two surplus doses. In theory, GPs could now prescribe guidelineappropriate quantities (as happens in other countries), but the associated breaking of packs may be resisted by pharmacists.

\section{Externally administered interventions}

Audit and feedback. Providing feedback to clinicians about their prescribing rates compared with the normative data of their peers can influence behaviour. ${ }^{20}$ Feedback based on PBS data is currently used by NPS MedicineWise as an intervention within a variety of national educational programs, including for antibiotic prescribing, but has not been trialled against controls. A variation of the method was trialled in the UK for high-prescribing GPs, who were sent a letter from the Chief Medical Officer, which resulted in a reduction in prescribing rates ${ }^{21}$ (Box 1). A similar mailing was sent by the Australian Commonwealth Officer recently. ${ }^{34}$

Academic detailing. Academic detailing comprises educational visits involving face-to-face education of prescribers by trained health care professionals. These visits can occur one to one or with small groups, and sometimes incorporate individual or practice level clinical and prescribing data. NPS MedicineWise has implemented national educational programs that include antibiotic prescribing.

\section{Activities that GPs can individually implement}

Delayed prescribing. In situations where the GP feels it is safe not to use antibiotics, but perceives that the patient (or parent) is anxious or feeling unsupported, a delayed prescription is an option. In this case, the GP writes an antibiotic prescription, but advises the patient not to have it dispensed unless some expected improvement does not take place or there is deterioration (described to the patient in detail). Evidence from a Cochrane review of randomised trials shows that this technique is effective at reducing antibiotic prescribing ${ }^{22}$ (Box 1 ).

Shared decision making. Australia lags behind the rest of the world in implementing this combination of communication skills and evidence-based practice. ${ }^{25,26,35}$ It is especially appropriate in decision making about antibiotic use for ARIs, where the benefits and harms are nearly balanced and, therefore, patient preferences become prominent. ${ }^{25}$ Trials of shared decision making for antibiotic use in ARIs show that this approach is effective at reducing antibiotic use $^{24}$ (Box 1). However, existing interventions are difficult for GPs to adopt, as few would afford the time and costs of learning how. Brief patient decision aids are a tool to support 
1 Interventions to support the reduction of antibiotic prescribing in Australian general practice

Intervention Details Effect size estimates Comment

\section{Regulatory interventions}

Repeats for Make the default "no repeat" prescriptions

antibiotics

Conserving some

antibiotics by

restricting access difficult to prescribe by using PBS restrictions (specific indications), or restricting some to authority to prescribe. However, introducing restrictions on a target antibiotic may result in increased use of unrestricted ones

Changing the packaging

Matching the dose (number of tablets or capsules) to guidelines for common indications

Antibiotic prescribing data are currently collected by PBS and fed back to GPs individually and confidentially, compared with norms of other peers

Personal letter to Letter from a senior government medical GPs from an doctor (eg, the Commonwealth Medical authoritative figure Officer) to high prescribers

\section{Activities that GPs can individually implement}

Delayed

prescribing

An adversarial relationship with the patient is avoided by offering prescriptions to patients who are inappropriately insistent on an antibiotic, but suggesting it should not be dispensed unless symptoms worsen or fail to improve after a specified time

Shared decision A combination of communication and evidencemaking based practice skills, in which the clinician explicitly evaluates the concerns, fears and expectations of the patient before detailing the benefits and harms of each management option (ie, antibiotics or none), and arriving at a shared decision that incorporates patient values and preferences ${ }^{25,26}$

"Nudge" techniques

Public declaration displayed in the practice of commitment to conserving antibiotics, together with supplemental information to patients

Voluntary audit Self-audit tools for the collection, feedback and and feedback activities reflection on management of respiratory tract infections $^{29}$

Medicinelnsight general practice reports on antibiotic prescribing in comparison with various indicators $^{15}$

\section{Supporting GPs' access to near-patient diagnostic testing}

A means of identifying patients at risk of serious bacterial infection

Procalcitonin

Streptococcal Serological test kits can detect whether group A A systematic review found the evidence

tests $\quad \beta$-haemolytic streptococcus is causing a sore throat contradictory ${ }^{32}$

\section{Public awareness campaigns}

Community education but likely to be small

There is evidence of generic effectiveness of a small

$18 \%$ reduction in antibiotic prescribing (in a

$20 \%$ reduction in inappropriate antibiotic prescribing, but from only a single RCT. ${ }^{28}$ This needs replicating in of $~ 19 \% 30$ antibiotics are not automatic), together with other intervention effects (ie, academic detailing antibiotic resistance, that not using antibiotics dissipates resistance and that antibiotics are not needed for common colds and influenza NPS MedicineWise already invests many resources in campaigns each winter
Untested, therefore unknown,

but likely to be small

Rates of fluoroquinolone use and resistance are low in Australia compared with equivalent countries (from ecological rates of use and resistance) $)^{17}$

Untested, therefore unknown increase (4-5\%) in clinician use of desired activity (more if the baseline performance is low and the audit and feedback is repeated), ${ }^{20}$ but has been trialled only among other interventions for antibiotic reduction

Currently used in several strategies in national educational programs

$3 \%$ decrease (95\% Cl, 2-4\%) if sent to $20 \%$ topprescribing practices. ${ }^{21}$ Whether repeating several times usefully increases the response is unknown

$62 \%$ (95\% Cl, 34-75\%) reduction in antibiotic prescribing (in a systematic review) ${ }^{22}$ systematic review) ${ }^{24}$

A trial is underway in Australia using a brief intervention (provision of patient decision aids) ${ }^{27}$ other settings

Unknown, and as yet untested in trials for antibiotic prescribing $^{20}$

A systematic review of observational and cluster randomised trials suggests a reduction in prescribin

Training is quite quick: $<1$ hour $^{31}$

A Cochrane review included two trials in primary care ( 1000 patients), which achieved a 40\% reduction in prescribing ${ }^{30,31}$

As an example, the French campaign Les antibiotiques c'est pas automatiques (ie, and supporting near-patient testing), reduced

Regulatory options are likely to produce a small but important reduction in prescribing

This access restriction can only affect expensive antibiotics (or GPs could simply prescribe privately to avoid authority to prescribe requirements)

Pharmacists may resist pack breaking

Implemented in Australia in 2017

Uptake very low in trials, but higher (13\%) in UK observationa studies $^{23}$

Existing interventions trialled are expensive to implement. Less lengthy interventions are needed to facilitate uptake by Australian GPs

GPs have to overcome only a few barriers to implement these techniques quickly

antibiotic prescribing by $>25 \%$, but the costs were high: $€ 500$ million
Costs can be very high to achieve broad reach and impact $^{33}$
All near-patient testing may add costs to the consultation, which would be difficult to ask either patients or their GPs to pay for 
shared decision making conversations and summarise and clearly communicate the evidence of benefits and harms of using and not using antibiotics. These decision aids may be effective and easy to implement $^{36}$ and are currently being trialled in Australia. ${ }^{27}$

"Nudge" techniques. These techniques are small behaviour changes that may deliver greater gains. In one randomised trial, GPs declared their commitment to antibiotic conservation in a signed poster with a picture of the GP displayed in the practice. Together with additional information available to provide to patients, this technique reduced antibiotic use ${ }^{28}$ (Box 1).

Voluntary audit and feedback activities. NPS MedicineWise has free electronic audit tools available to GPs to collect feedback and reflect on their management of ten consecutive patients with respiratory tract infections. More rigorously, GPs in practices participating in the MedicineInsight program, in which data are routinely extracted and de-identified from electronic patient records, have access to more detailed reports about their prescribing in comparison to their peers. ${ }^{15}$ This access to data reduces any confounding of changing diagnostic thresholds that bedevil documenting the management of ARIs. ${ }^{37}$

Symptom management. Practical advice on managing symptoms may be offered as an alternative to prescribing antibiotics, which may also manage the patient's expectation of a prescription (ie, "filling the therapeutic vacuum").

\section{Supporting GPs' access to near-patient diagnostic testing}

One of the concerns GPs face is diagnostic uncertainty. Although almost all ARIs are safe to manage with expectant observation, every GP knows that in the next patient presenting with an ARI, the condition may be the precursor to or the early stages of a more sinister illness, such as meningococcal meningitis, communityacquired pneumonia, peritonsillar abscess, mastoiditis and even the non-suppurative complications of acute rheumatic fever, or acute glomerulonephritis. Clinical decisions can be supported by near-patient testing and diverse methods, such as streptococcal tests, C-reactive protein and procalcitonin, have been trialled with mixed success ${ }^{30,31}$ (Box 1). Some countries (eg, Denmark) have invested in this technology wholesale. However, the acceptability to clinicians and patients has been poorly studied in Australia, and it is uncertain whether there would be political support for an increase in the cost of GP consultations - which may about double with near-patient testing — when antibiotics remain inexpensive.

\section{Public awareness campaigns}

Community education is essential in supporting the shift of GPs' attention to reduced antibiotic prescribing. One concern is that GPs feel pressure to prescribe because of their perception that many patients expect antibiotics more often than is indicated, believing that antibiotics reduce symptom duration more than they actually do. ${ }^{39}$ GPs place a high value on rapport with their patients - for clinical, as well as financial reasons, in open-access and feefor-service primary care. This perception may often be wrong a good reason for employing shared decision making — but, nonetheless, some patients certainly expect antibiotics more often than is realistic. NPS MedicineWise has conducted several consumer campaigns to change these community expectations, using simple advertising and innovative social media campaigns (http:// i2p.com.au/nps-media-releases-antibiotic-resistance). A social marketing approach has been used, including strategies that leverage collectivism, use a social norms approach, nudge theory, gamification, celebrity endorsement and cocreation. Consumer campaigns have been implemented mainly during winter, and include the use of traditional media, social media, television (including in GP waiting rooms), magazines, newspapers, outdoor advertising and short film competitions. A sustained, concerted, effort on public awareness is needed to effect change. ${ }^{33,40}$

\section{Unanswered questions}

In addition to the interventions described, more research is necessary to answer a myriad of unanswered questions (Box 2). Many of the interventions listed need testing in Australia's fee-forservice environment. In particular, we need to understand the passage of antibiotic resistance from patients treated in the community to where this resistance does the most harm: in hospital

\section{Unanswered questions to further inform strategies in Australian general practice to reduce antibiotic resistance}

\section{Sets of unanswered questions}

What is the relationship between antibiotic use and the development of antibiotic resistance?

How does this change with different antibiotics used?

What are the effects of antibiotics against common uncomplicated skin infections (eg, cellulitis) and urinary tract infections (eg, in pre-menopausal women)

Are interventions that are effective in trials conducted in international settings (Box 1) as effective in Australia?

What are the effective symptom management alternatives to antibiotics?

What approaches should be taken to deal with overprescribing of antibiotics in residential aged care facilities?

How unanswered questions may inform strategies to reduce antibiotic resistance

A central assumption in the imperative to reduce prescribing is that use causes resistance. This has shown to be true for some antibiotics and some infections. ${ }^{6}$ However, use does not always induce resistance (eg, penicillin for the group A streptococcus of sore throats) ${ }^{6}$

In contrast to the evidence for acute respiratory infections, which is substantial, there are few trials of antibiotics against placebo for skin and soft tissue infections, or urinary tract infections ${ }^{41,42}$ These two indications are also common reasons for antibiotic use in primary care, and being able to quantify the benefits and harms would enable better decision making

In Australia, there are some specific health service characteristics (eg, fee-for-service funding of primary care, a disconnection in funding for primary care and hospital services, and lack of patient registration to general practices) that may affect the effectiveness of approaches trialled in other countries

There is a long list of such potentially effective treatments, ranging from simple analgesics and systemic steroids to complementary and alternative medicines. The evidence is sparse on evaluating and quantifying their effects ${ }^{43,44}$

Studies have uncovered a very serious problem in some residential aged care facilities, with, for example, $40 \%$ of antibiotics being used without evidence of infection. There are specific challenges in caring for patients in these settings, with increased difficulties in making diagnoses, multiple carers and pathology services. The problems appear to be very different from mainstream general practice, with several workforce, logistical and cultural factors ${ }^{45}$ 
care. ${ }^{2}$ What alternatives can be suggested to fill the "therapeutic vacuum" left by not offering antibiotics?

So far, the focus has properly been on ARIs because these are the conditions for which most antibiotics are prescribed. But another reason is that, historically, ARIs have the most evidence - in the form of randomised placebo controlled trials - about the benefits and harms of antibiotic use. For the two other major groups of indications for antibiotics in general practice (ie, skin and soft tissue infections and urinary tract infections), there are very few placebo controlled trials. There is some evidence that uncomplicated urinary tract infections in pre-menopausal women may be treated almost as effectively with non-steroidal anti-inflammatory drugs. ${ }^{41,42}$ But in the main, GPs currently need to resort to pathophysiological and microbiological information to estimate the effect that antibiotics will have - an approach that led to overprescribing for ARIs in the past, until systematically reviewed placebo randomised trials showed that antibiotics have small benefits.

\section{Conclusions}

Extrapolating from the initiatives in other countries to decrease antibiotic usage and their impact on resistance, it is highly likely that reducing antibiotic use in Australian general practice will decrease antibiotic resistance. Therefore, it seems prudent to start practising antibiotic conservation now to proceed in parallel with any future new antibiotic class development.

There are two notable characteristics about the interventions described in this review. The first one is the diversity of potential interventions. We cannot be sure how their effects interact, but their different modes of action suggest that they are likely to be additive and possibly synergistic. Second, the effects accumulate slowly over the years. Data from Sweden, which has had a concerted drive against antibiotics in general practice to become one of the lowest antibiotic prescribers in the world, show that the gains are incremental, a steady few percentage reduction over many years. ${ }^{5,46}$ The Medical Research Future Fund has prioritised an initial \$5.9 million to support antimicrobial resistance research, ${ }^{47}$ although what proportion of this fund will focus on reducing antibiotic use in the community remains to be seen. Strategies in Australia will require appropriate resources and implementation support for employing multiple modalities and a commitment for a sustained effort.

Acknowledgements: We received funds from the National Health and Medical Research Council for the Centre for Research Excellence in Minimising Antibiotics in Acute Respiratory Infections in Primary Care.

Competing interests: We have been commissioned by the Australian Commission for Safety and Quality and Health Care and Bupa to provide expertise and to design patient decision aids.

Provenance: Not commissioned; externally peer reviewed.

(c) 2017 AMPCo Pty Ltd. Produced with Elsevier B.V. All rights reserved.
1 World Health Organization. Antimicrobial resistance: global report on surveillance, 2014. Geneva: WHO; 2014 http://www.who.int/drugresistance/documents/ surveillancereport/en (accessed May 2017).

2 O'Neill J, Chair. Antimicrobial resistance: tackling a crisis for the health and wealth of nations. London: HM Government (UK) and Wellcome Trust; 2014. http:// amr-review.org/sites/default/files/AMR\%20Review \%20Paper\%20-\%20Tackling\%20a\%20crisis\%20for \%20the $\% 20$ health\%20and\%20wealth\%20of \%20nations_l.pdf (accessed Sept 2017).

3 Australian Commission on Safety and Quality in Health Care. AURA 2016: first Australian report on antimicrobial use and resistance in human health. Sydney: ACSQHC; 2016. https: //www.safetyandquality.gov.au/publications/ aura-2016-first-australian-report-on-antimicroibal-useand-resistance-in-human-health (accessed Apr 2017).

$4 \mathrm{Høg} B B$, Korsgaard H, Sönksen UW, editors. DANMAP 2015 - use of antimicrobial agents and occurrence of antimicrobial resistance in bacteria from food animals, food and humans in Denmark. Danish Integrated Antimicrobial Resistance Monitoring and Research Programme; 2015. http://www.danmap.org/ /media/ Projekt\%20sites/Danmap/DANMAP\%2Oreports/ DANMAP\%20\%202015/DANMAP\%202015.ashx (accessed May 2017).

5 Tyrstrup M, Beckman A, Mölstad S, et al. Reduction in antibiotic prescribing for respiratory tract infections in Swedish primary care- a retrospective study of electronic patient records. BMC Infect Dis 2016; 16: 709.

6 Costelloe C, Metcalfe C, Lovering A, et al. Effect of antibiotic prescribing in primary care on antimicrobial resistance in individual patients: systematic review and meta-analysis. BMJ 2010; 340: c2096.

7 Fletcher-Lartey S, Yee M, Gaarslev C, Khan R. Why do general practitioners prescribe antibiotics for upper respiratory tract infections to meet patient expectations: a mixed methods study. BMJ Open 2016; 6: e012244

8 Craig JC, Williams GJ, Jones M, et al. The accuracy of clinical symptoms and signs for the diagnosis of serious bacterial infection in young febrile children: prospective cohort study of 15781 febrile illnesses. BMJ 2010; 340.

9 Butler CC, Rollnick S, Pill R, et al. Understanding the culture of prescribing: qualitative study of general practitioners' and patients' perceptions of antibiotics for sore throats. BMJ 1998; 317: 637-642.

10 Hoffmann TC, Del Mar C. Clinicians' expectations of the benefits and harms of treatments, screening, and tests: A systematic review. JAMA Int Med 2017; 177: 407-419.

11 Coombs G, Pearson J, Nimmo G, Christiansen K. Molecular epidemiology of MRSA in the Australian community; AGAR SAPIO Conference; Brisbane 2012 http://www.agargroup.org/publications (accessed Sept 2017).

12 Del Mar C. Managing sore throat: a literature review. I. Making the diagnosis. Med J Aust 1992; 156: 572-575.

13 Britt $\mathrm{H}$. Bettering the evaluation and care of health (BEACH); media statement: closure of BEACH program after 18 years [website]. Sydney: University of Sydney; 2016. http://sydney.edu.au/medicine/fmrc/media/ BEACH-closure-2016-04.php (accessed May 2017).

14 Dallas A, Magin P, Morgan S, et al. Antibiotic prescribing for respiratory infections: a cross-sectional analysis of the ReCEnT study exploring the habits of early-career doctors in primary care. Fam Pract 2015; 32: 49-55.

15 NPS MedicineWise. Using Medicinelnsight data: Australia's first large-scale general practice data program [website]. Sydney: NPS MedicineWise; 2016. https://www.nps.org.au/medicine-insight/usingmedicineinsight-data (accessed June 2017).

16 McCullough AR, Pollack AJ, Plejdrup Hansen M, et al. Antibiotics for acute respiratory infections in general practice: comparison of prescribing rates with guideline recommendations. Med J Aust 2017; 207: 65-69. https:// www.mja.com.au/journal/2017/207/2/antibiotics-acuterespiratory-infections-general-practice-comparisonprescribing

17 Cheng AC, Turnidge J, Collignon P, et al. Control of fluoroquinolone resistance through successful regulation, Australia. Emerg Infect Dis 2012; 18: 1453-1460.
18 McGuire TM, Smith J, Del Mar C. The match between common antibiotics packaging and guidelines for their use in Australia. Aust N Z J Public Health 2015; https:// doi.org/10.1111/1753-6405.12385.

19 eTG complete Melbourne Antibiotics 2017 Therapeutic Guidelines Limited Antibiotic version 15. http://www.tg. org.au/index.php?sectionid=41 (accessed June 2017).

20 Ivers N, Jamtvedt G, Flottorp S, et al. Audit and feedback: effects on professional practice and healthcare outcomes. Cochrane Database Syst Rev 2012; (6): CD000259.

21 Hallsworth M, Chadborn T, Sallis A, et al. Provision of social norm feedback to high prescribers of antibiotics in general practice: a pragmatic national randomised controlled trial. Lancet 2016; 387: 1743-1752.

22 Spurling GK, Del Mar CB, Dooley L, et al. Delayed antibiotics for respiratory infections. Cochrane Database Syst Rev 2013; (4): CD004417. (Update in press 2017).

23 Little P, Stuart B, Smith S, et al. Antibiotic prescription strategies and adverse outcome for uncomplicated lower respiratory tract infections: prospective cough complication cohort (3C) study. BMJ 2017; 357.

24 Coxeter P, Del Mar Chris B, McGregor L, et al. Interventions to facilitate shared decision making to address antibiotic use for acute respiratory infections in primary care. Cochrane Database Syst Rev 2015; (11): CD010907.

25 Hoffmann TC, Légaré F, Simmons MB, Shared decision making: what do clinicians need to know and why should they bother? Med J Aust 2014; 201: 35-39. https://www.mia.com.au/journal/2014/201/1/shareddecision-making-what-do-clinicians-need-know-andwhy-should-they-bother

26 Trevena L, Shepherd HL, Bonner C, et al. Shared Decision Making in Australia in 2017. Zeitschrift für Evidenz, Fortbildung und Qualität im Gesundheitswesen 2017; 123-124: 17-20.

27 Hoffmann TC, Del Mar CB. Effect of decision aids for acute respiratory infections on the use of antibiotics in 
general practice: a cluster randomised controlled trial. Trial ID no. ACTRN12616000644460. Australian New Zealand Clinical Trials Registry; 2016. https://www. anzctr.org.au/Trial/Registration/TrialReview.aspx?id=37 0698\&isReview=true (accessed Aug 2017).

28 Meeker D, Knight TK, Friedberg MW, et al. Nudging guideline-concordant antibiotic prescribing: a randomized clinical trial. JAMA Inter Med 2014; 174: 425-431.

29 NPS MedicineWise. Management of specific respiratory tract infections: clinical e-audit. Sydney: NPS MedicineWise; 2017. https://www.nps.org.au/cpd/ activities/management-of-specific-respiratory-tractinfections? $p=$ GPs (accessed May 2017).

30 Huang $\mathrm{Y}$, Chen R, Wu T, et al. Association between point-of-care CRP testing and antibiotic prescribing in respiratory tract infections: a systematic review and meta-analysis of primary care studies. $\mathrm{Br} J$ Gen Pract 2013; 63: e787-e794.

31 Cals JW, Butler CC, Hopstaken RM, et al. Effect of point of care testing for $\mathrm{C}$ reactive protein and training in communication skills on antibiotic use in lower respiratory tract infections: cluster randomised trial. BMJ 2009; 338: bl374.

32 Delaney BC, Hyde CJ, McManus RJ, et al. Systematic review of near patient test evaluations in primary care. BMJ 1999; 319: 824-827.

33 MacReady N. French campaign reduces antibiotic prescriptions. Lancet Infect Dis 2009 9: 469.

34 Wright J. Thousands of GPs rapped for high antibiotic prescribing. Australian Doctor (Sydney) 2017; 27 June. https://www.australiandoctor.com.au/news/latestnews/thousands-of-gps-rapped-for-high-antibioticprescr (accessed Aug 2017).

35 Hoffmann TC, Montori VM, Del Mar C. The connection between evidence-based medicine and shared decision making. JAMA 2014; 312: 1295-1296.

36 Coxeter PD, Del Mar CB, Hoffmann TC. Preparing parents to make an informed choice about antibiotic use for common acute respiratory infections in children: a randomised trial of brief decision aids in a hypothetical scenario. The Patient 2017; 10: 463-474.

37 Howie JG, Richardson IM, Gill G, Durno D. Respiratory illness and antibiotic use in general practice. J R Coll Gen Pract 1971; 21: 657-663.

38 NPS MedicineWise. Respiratory tract infections: manage your symptoms. Sydney: NPS MedicineWise; 2016. https://activities.nps.org.au/nps-order-form/Resources/ NPS-MedicineWise-RTI-Action-Plan.pdf (accessed June 2017).

39 Coxeter PD, Del Mar CB, Hoffmann TC. Parents' expectations and experiences of antibiotics for acute respiratory infections in primary care. Ann Fam Med 2017; 15: 149-154.

40 Sabuncu E, David J, Bernède-Bauduin C, et al. Significant reduction of antibiotic use in the community after a nationwide campaign in France, 2002-2007. PLoS Med 2009; 6: el000084.

41 Bleidorn J, Gagyor I, Kochen M, et al. Symptomatic treatment (ibuprofen) or antibiotics (ciprofloxacin) for uncomplicated urinary tract infection? Results of a randomized controlled pilot trial. BMC Med 2010; 8: 30.

42 Gagyor I, Bleidorn J, Kochen MM, et al. Ibuprofen versus fosfomycin for uncomplicated urinary tract infection in women: randomised controlled trial. BMJ 2015; 351: h6544.

43 Thomas M, Del Mar C, Glasziou P. How effective are treatments other than antibiotics for acute sore throat? $\mathrm{Br} J$ Gen Pract 2000: 50: 817-820.

44 Hayward G, Thompson MJ, Henegan CJ, et al. Corticosteroids for relief of pain in sore throat: a systematic review and meta-analysis. BMJ 2009; 339 : 2976.

45 Lim CJ, Kwong MW, Stuart RL, et al. Antibiotic prescribing practice in residential aged care facilities health care providers' perspectives. Med J Aust 2014; 201 98-102. https://www.mia.com.au/journal/2014/201/2/ antibiotic-prescribing-practice-residential-aged-carefacilities-health-care

46 Public Health Agency of Sweden and National Veterinary Institute. Use of antimicrobials and occurrence of antimicrobial resistance in Sweden. UppaL, Sweden: SWEDRES/SVARM; 2013. http://www.sva.se/ globalassets/redesign2011/pdf/om_sva/publikationer/ swedres_svarm2013.pdf (accessed June 2017).

47 Australian Department of Health. Investing $\$ 5.9$ million to tackle antimicrobial resistance [media release]. 26 May 2017. http://www.health.gov.au/internet/ministers/ publishing.nsf/Content/health-mediarel-yr2017hunt054.htm (accessed June 2017). 